\title{
Audit of patients' awareness of ophthalmic diagnoses
}

\author{
Sudha Sudesh, Susan M Downes, Peter J McDonnell
}

\begin{abstract}
Providing information to patients about their medical condition and treatment options is important in medical management. To assess patients' knowledge of their ocular disease, prognosis, and treatment a questionnaire based survey was performed. 219 patients selected by random systematic sampling during six months from patients attending general ophthalmic clinics in Selly Oak Hospital, Birmingham, were questioned and 217 questionnaires were analysed. The findings showed that patients' knowledge of their diagnosis depended on their condition: patients with common conditions such as glaucoma and cataract had a better understanding of their condition, its treatment, and prognosis compared with patients with rarer conditions such as retinal detachment or patients with multiple diagnoses. In all, 152 patients $(70 \%)$ wanted more information about their condition; $49(23 \%)$ did not (although $12(25 \%)$ had attempted to obtain information); and $16(7 \%)$ were undecided. In view of the few patients with a precise understanding of their ophthalmic diagnosis and prognosis and the majority's wish for access to further information, that access needs improvement and different modes of disseminating the information should be implemented. (Quality in Health Care 1993;2:175-178)
\end{abstract}

\section{Introduction}

An increasingly important part of medical management is the adequate and appropriate provision of information to patients about their medical condition and the management options available. When patients' views have been sought to help in assessing the quality of health care delivery one recurrent complaint is identified: doctors seldom provide sufficient information to patients about their medical problems. ${ }^{12}$ This project was designed to investigate patients' awareness of their ophthalmic diagnosis, the prognosis, and long term implications. We did not assess the methods and quality of previously imparted information but aimed at establishing a baseline of patient awareness at the time of the audit. We also looked at the need for aids to information.

\section{Patients and methods}

The survey was carried out in general ophthalmology clinics in Selly Oak Hospital, Birmingham, from October 1990 to April 1991. A pilot survey was done on 10 patients to assess the clarity and acceptability of the questionnaire (box). This consisted of six questions related to understanding the ocular diagnosis, prognosis, and treatment. Patients were then asked whether they would like access to further information. Background variables, including social and demographic data were also recorded.

Patients were recruited into the survey by random systematic sampling of routine ophthalmic outpatient lists. During the six months of the survey every fifth patient seen in the clinic was assessed. After oral consent had been given the assessment was made by means

\section{Patient questionnaire}

Hospital No:

Sex:

Date of birth:

Ethnic origin:

Profession:

1 Do you know what is the problem with your eyes?

2 Do you know what that means?

3 Do you know what will happen in the long term?

4 Do you know what treatment you are receiving? What is the dosage?

5 Do you know what to do if you run out of medication?

6 Do you think a simple leaflet (cassette) explaining your condition and treatment will be useful to you?

Diagnosis:

Treatment:

Information:

Review since:
Ms Sudesh

Accepted for publication 25 April 1993 


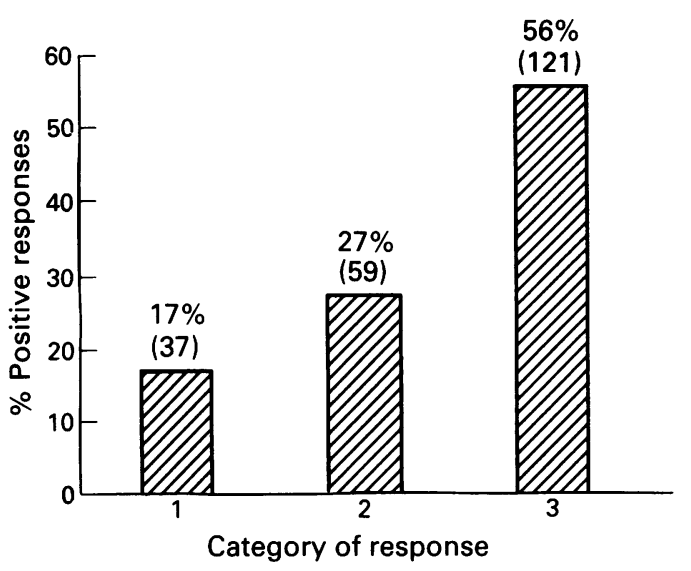

Fig 1 Percentage (number) of 217 patients able to name diagnosis, by category of response (1 does not know name, 2 does not know full name (s) of all diagnoses, 3 knows complete medical name)

of an interview based on the questionnaire. The questionnaire was put to the patients by SS or SMD before the outpatient consultation. The patients' notes were subsequently reviewed jointly by the authors and details of diagnosis, treatment, and total duration of medical review in the clinic were recorded. The patients' responses were categorised to reflect the degree of understanding.

\section{Results}

A total of 219 patients was surveyed. The responses of two patients were excluded because of the patients' mental state, leaving 217 questionnaires for analysis. Patients' age ranged from 21 to 91 years; 42 patients were aged under 60 years, 48 were aged 60-69 years, 81 aged $70-79$ years, and 46 were aged 80 years and over. Ninety eight patients were male and 119 female.

Knowledge of medical name of diagnosis

Responses to the first question, which related to knowledge of the medical name of the diagnosis showed that although $56 \%(121 / 217)$ patients knew the correct name for their condition, the rest either did not know the

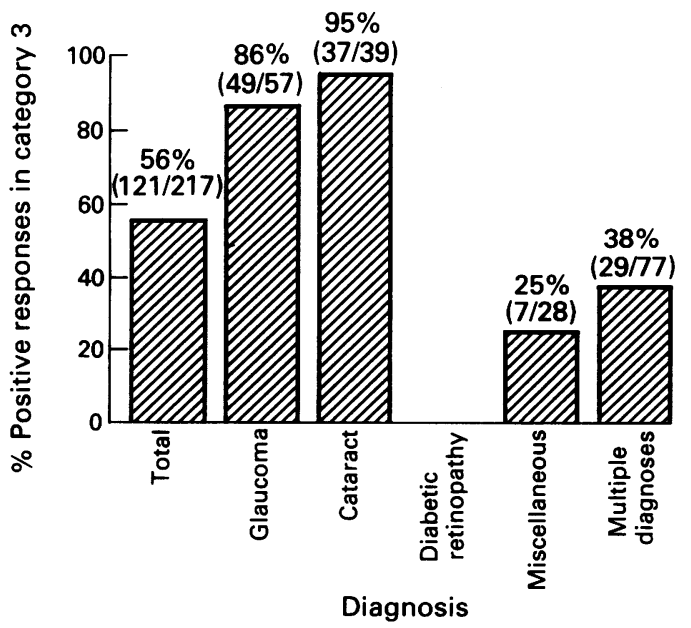

Fig 2 Percentage (number) of patients giving responses in category 3 (knows complete medical name), according to patient's diagnosis

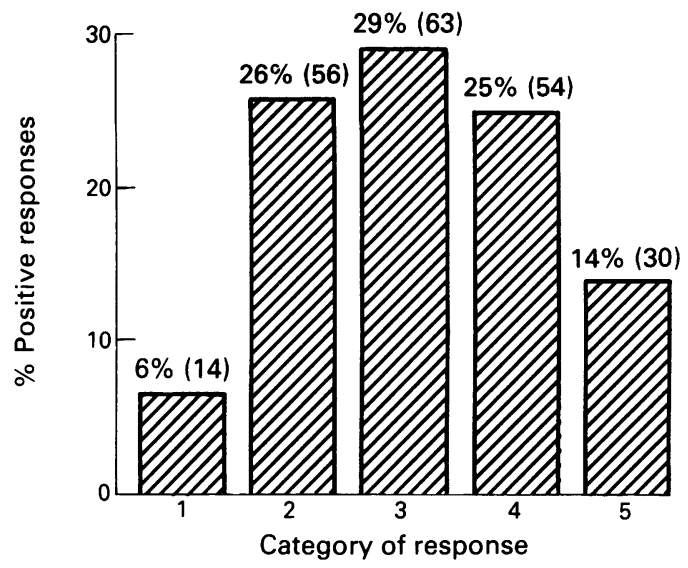

Fig 3 Percentage (number) of 217 patients understanding diagnosis and prognosis of condition, by category of response (1 knows nothing; 2 knows only diagnosis; 3 knows diagnosis and prognosis, no understanding of pathology; 4 good understanding, does not know everything; 5 knows everything)

name at all or knew it incompletely (fig 1). Patients with common conditions were more likely to be able to name their diagnosis than those with less common conditions or multiple diagnoses. Most patients with glaucoma ( $86 \%$, $49 / 57)$, or cataract $(95 \%, 37 / 39)$ knew their diagnosis, but only $25 \%(7 / 28)$ of patients knew the names of miscellaneous other conditions, and none of 16 diabetic patients questioned knew the term diabetic retinopathy (fig 2).

\section{Understanding the diagnosis}

The responses to the first three questions, which concerned diagnosis and prognosis, were grouped together and categorised using a system which took into account knowledge of the diagnosis, elementary pathology, and prognosis (fig 3). Only $14 \%(30 / 217)$ of patients knew everything about their condition, and over half of those surveyed were unaware of the implications of their diagnosis. Once again, those who had multiple diagnoses or rarer disorders were less likely to understand the problem than those with common conditions such as cataract and glaucoma (fig 4).

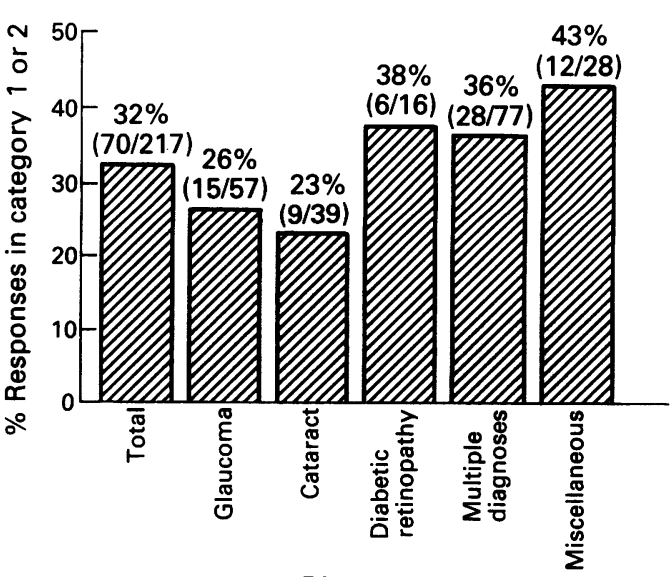

Diagnosis

Fig 4 Percentage (number) of patients giving responses in category 1 or 2 (1 knows nothing, 2 knows only diagnosis), according to patient's diagnosis 
Understanding drug treatment for ocular problems

In total, 132 patients were taking drugs for their ocular condition. Responses to questions 4 and 5, which asked about drug treatment, were grouped into five categories according to overall knowledge about the name, strength, and daily dose of the drug and awareness of

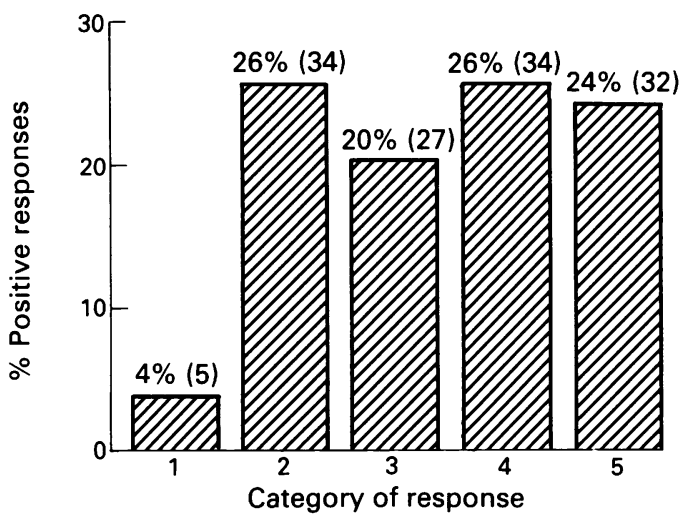

Fig 5 Percentage (number) of 132 patients receiving drug treatment understanding their treatment, by category of response (1 knows nothing; 2 knows one item of: name, strength, dosage of drug and need for GP's repeat prescription; 3 knows two items; 4 knows three; 5 knows everything)

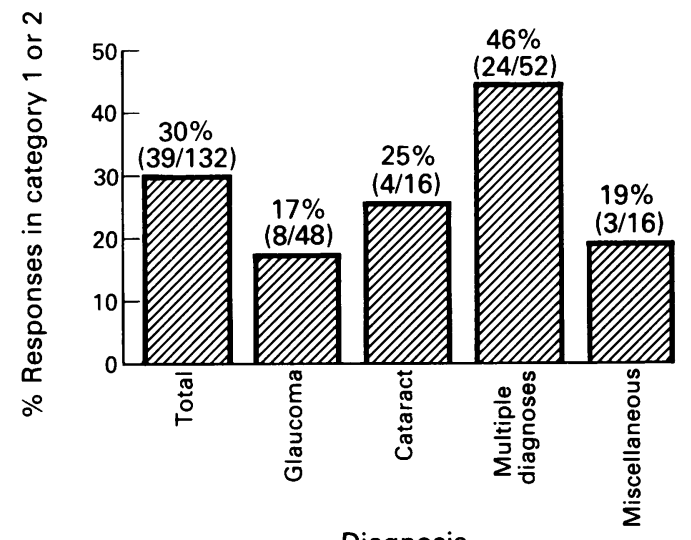

Diagnosis

Fig 6 Percentage (number) of patients giving responses in category 1 or 2 (knows nothing, knows one item), according to patient's diagnosis

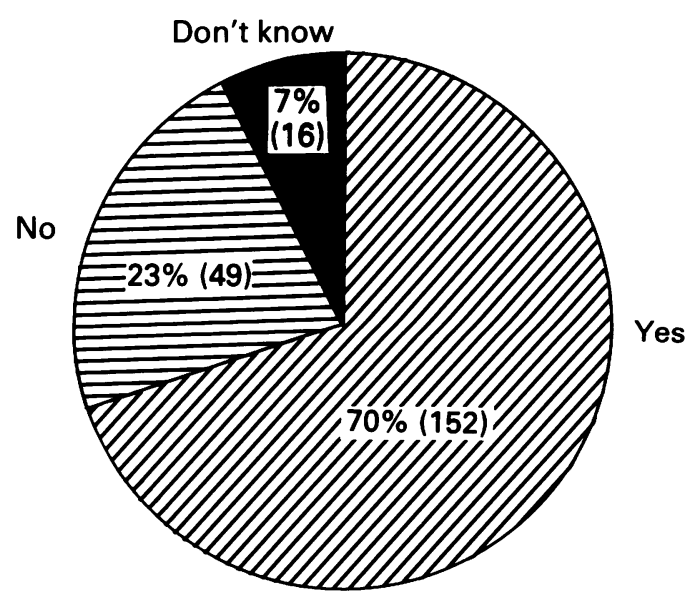

Fig 7 Patients' response to need for further information $(n=217)$ the need or facility for renewing the prescription by the general practitioner (fig 5). Patients being treated for multiple ocular problems were less likely to understand details of their treatment than those with single common problems (fig 6).

\section{Aids to information}

Of all 217 patients, $152(70 \%)$ wanted more information about their ocular condition, $49(23 \%)$ did not, and $16(7 \%)$ were undecided (fig 7). Of the 49 who declined further information, $12(25 \%)$ had already made their own efforts to obtain more information.

\section{Discussion}

Apparently, although over half of these patients knew the correct terminology for their diagnosis, few had a precise understanding of their condition. Patients who had common ocular diseases, such as cataract or glaucoma, had a better understanding than those whose ocular condition was less common - for example, retinal detachment, herpes simplex keratitis, keratoconus, dry eyes, retinal vein occlusion, and ocular myasthenia. Perhaps not surprisingly, patients with more than one concurrent ocular problem understood their problem less well than those with single, common problems, as they have more information to retain. This pattern was seen also in responses to the questions about ocular drug treatment; patients' knowledge of their treatment regimens was better for those with common conditions than the rest. Most respondents wanted access to more information.

It is important to find out what patients understand of their disease as motivation to comply with treatment depends on the patients' understanding of their complaint. ${ }^{34}$ We have illustrated the need for improved provision of information in ophthalmology clinics, particularly to patients with rarer conditions or multiple conditions. Explanation of the condition and ensuring the patient's understanding will improve patient participation in managing their disease. Our questionnaire highlighted certain areas of misunderstanding and showed that many of our patients are underinformed and lack knowledge about their ocular problems. It would be difficult to determine what information had actually been given to the patient, but a simple repetition of the facts from doctor to patient seems unlikely to ensure comprehension of the medical condition. It was not within the scope of this study to assess patient's ability to understand the information presented to him or her.

In considering ways of delivering more information, two issues arise: how well is the doctor equipped to give that information and what is the best method of transferring that information to the patient? Much attention has been given recently to educating doctors in communication skills as part of medical undergraduate training. There should also be regular updates at a postgraduate level. The role of other members in the health care team 
in patient education also needs exploring. The target audience can be reached in several ways. A quick, inexpensive means of imparting information in the form of simple information leaflets can reach most patients. For those whose eyesight precludes the use of visual aids, prerecorded cassettes are useful. Other more expensive equipment including video recordings could be kept for use in the clinic itself after a consultation with the doctor. At present the main method of disseminating information is to repeat the salient features of the condition and the necessity for treatment at each visit.
A future project would be to assess the impact of distributing information leaflets to patients to evaluate their response to the information in terms of understanding and satisfaction.

1 Cartwright A. Human relations and hospital care. London: Routledge and Kegan Paul, 1962.

2 Gregory J. Patients' attitudes to the hospital service. London: HMSO, 1978.

3 Kinsey J, Bradshaw P, Ley P. Patients' satisfaction and report of acceptance of advice in general practice. $\mathfrak{f} R$ Coll Gen Pract 1975;25:558-66.

4 Salmon P, Quine J. Patients intentions in primary care: measurement and preliminary investigation. Psychology and Health 1989;3:103-10. 